\title{
CDISC SEND Test Article Physical Substance Classification Response Terminology
}

National Cancer Institute

\section{Source}

National Cancer Institute. CDISC SEND Test Article Physical Substance Classification

Response Terminology. NCI Thesaurus. Code C154683.

Terminology associated with the test article physical substance classification response codelist of the Clinical Data Interchange Standards Consortium (CDISC) Standard for the Exchange of Non-clinical Data (SEND). 